\title{
Las Universidades Españolas y EEES: Un Estudio Sobre los Títulos de Grado de Maestro en Educación Primaria
}

\author{
Laura Jiménez $^{(1)}$, Francisco J. Ramos ${ }^{(2)}$ y Mercedes Ávila ${ }^{(3)}$ \\ Universidad de Castilla-La Mancha, Facultad de Educación, (1) Departamento de Psicología, \\ (2) Departamento de Pedagogía, (3) Departamento de Filosofía (Área de Sociología), \\ Campus Universitario s/n, 16071 Cuenca-España (e-mail: Laura.Jimenez@uclm.es; \\ FranciscoJ.Ramos@uclm.es; Mercedes.Avila@uclm.es)
}

Recibido Ago. 11, 2011; Aceptado Oct. 06, 2011; Versión final recibida Oct. 21, 2011

\begin{abstract}
Resumen
El objetivo de esta investigación es analizar las diferencias existentes entre doce universidades españolas en la formación inicial de los maestros. Específicamente, el estudio se centra, en primer lugar, en los objetivos y competencias del título y, en segundo lugar, en la planificación de enseñanzas desarrollada por cada universidad. Para ello, se analizaron las memorias de verificación de los títulos de maestro de primaria de 12 universidades españolas, verificadas previamente por La Agencia Nacional de Evaluación de la Calidad y Acreditación (ANECA). Los resultados preliminares muestran que existen grandes diferencias entre los objetivos y las competencias propuestas por las doce universidades. También se han encontrado diferencias significativas en el número y tipo de menciones ofertadas por cada una de ellas, así como en la distribución de las prácticas en los distintos periodos académicos.
\end{abstract}

Palabras clave: Espacio Europeo de Educación Superior; memorias de verificación ANECA; objetivos y competencias; planificación de enseñanzas; Grado de Maestro en Educación Primaria

\section{The Spanish Universities and the European Higher Education Area: A Study on the Degree Title of Primary Education}

\begin{abstract}
The aim of this paper is to analyze the differences between twelve Spanish universities concerning the initial training of Primary Education teachers. Specifically, the study focuses, firstly, on the objectives and competences aimed at the Degree title of Primary Education and, secondly, on the teaching-learning programs developed by each university. To carry out this study, 12 Spanish universities' verification guides (ANECA), covering the Degree program for Primary School Teacher, were analyzed. Preliminary results show that there are major differences between the twelve universities since the objectives and competences proposed in their learning programs are different. There are also significant differences in type of qualification modules offered by the twelve universities and their distribution of the teaching practices throughout the different academic courses.
\end{abstract}




\section{INTRODUCCIÓN}

A pesar de la falta de información y las reticencias por parte del profesorado universitario con relación a la transformación de las enseñanzas universitarias (Fernández Díaz et al, 2010), la reforma de los estudios universitarios en España para su adaptación al Espacio Europeo de Educación Superior (EEES) es hoy una realidad. Antes iniciar dicha transformación, las universidades españolas habían estado reguladas por la Ley Orgánica 11/1983, de 25 de agosto, de Reforma Universitaria (LRU). Esta ley permitió una mayor democratización interna y la conquista de la autonomía universitaria necesaria tras el periodo de dictadura franquista. Esto derivó en el incremento de las relaciones académicas y científicas con otras instituciones de educación europeas, en las que la cultura de la calidad ya estaba bastante instalada.

En 1988, en Bolonia, casi 400 universidades firmaban la Carta Magna para proclamar y defender la autonomía universitaria y la libertad académica, señalar la necesidad de reconocer ciertas garantías de los estudiantes, así como potenciar la selección del profesorado y el intercambio entre universidades. Diez años después, a mediados de 1998, comenzaban a debatirse el futuro de la universidad y el modelo de educación superior deseados para Europa. El 19 de junio de 1999, 30 ministros de educación europeos firmaban la Declaración de Bolonia que sentaba las bases para la construcción de un área de educación superior integrada. El proceso de creación de este espacio es conocido desde entonces como Proceso de Bolonia. Se pretendía conseguir, entre otros, la adopción de un sistema fácilmente legible y comparable de titulaciones, así como la promoción de la movilidad de alumnos, profesores y personal administrativo entre las instituciones de enseñanza superior europea.

En España, durante este periodo, se comenzaba a reflexionar sobre las transformaciones a llevar a cabo en política educativa y en relación a las distintas estructuras y contenidos universitarios. Ésta fue la finalidad del informe "Universidad 2000", elaborado por encargo de la Conferencia de Rectores de las Universidades Españolas (CRUE) durante el año 1999, en el que se defendía un cambio profundo en la universidad en España, en Europa y, en general, en todo el mundo. Una de sus muchas conclusiones fue la convicción de que era necesario superar los obstáculos para la movilidad, la armonización y la apuesta por la calidad. Un año después, tras una gran polémica y oposición por parte de una gran mayoría de la comunidad universitaria, en diciembre de 2001 se aprobaba la Ley Orgánica 6/2001, de 21 de diciembre, de Universidades (LOU), sustituyendo y derogando a la LRU. Precisamente, uno de los objetivos era "mejorar la calidad docente, investigadora y de gestión de la universidad" e "integrarse competitivamente junto a los mejores centros de enseñanza superior en el nuevo espacio universitario europeo que se está comenzado a configurar". Entre 2001 y 2002, se daba un nuevo paso hacia la integración de la educación superior europea con el desarrollo del proyecto de investigación europeo Tuning Educational Structures in Europe, conocido posteriormente como Proyecto Tuning. Este proyecto piloto estaba encaminado a facilitar la convergencia en la educación superior en Europa, desarrollar perfiles profesionales, resultados de aprendizaje y competencias deseables y facilitar la transparencia en las estructuras educativas.

En España, y con el propósito de mejorar la calidad del sistema universitario y adecuar la estructura de las enseñanzas españolas al EEES, se fundaba en 2002 la Agencia Nacional de Evaluación de la Calidad y Acreditación (ANECA). Una de las funciones de ANECA era establecer y publicar los procedimientos, protocolos y guías para la verificación de los títulos oficiales adaptados al EEES, así como evaluar las propuestas de planes de estudio. Para ello, mediante el programa denominado VERIFICA, las universidades se sometían voluntariamente a un proceso de evaluación las memorias en las que se desarrollan los planes de estudio de los títulos universitarios. En la actualidad, la mayoría de las universidades ha finalizado con dicha evaluación. Sin embargo, no será hasta la aparición de la Ley Orgánica 4/2007, de 12 de abril, por la que se modifica la Ley Orgánica de Universidades (LOMLOU), cuando se termine de configurar una reforma universitaria española que iba a permitir continuar el proceso de convergencia hacia el EEES, potenciando la autonomía de las universidades, aumentando la rendición de cuentas sobre el cumplimiento de sus funciones, y reestructurando y organizando las enseñanzas universitarias en base a tres ciclos: Grado, Máster y Doctorado. Asimismo, con esta nueva 
organización se incorpora la garantía de calidad como uno de los elementos básicos que un plan de estudios debe contemplar como uno de los elementos imprescindibles de las futuras propuestas de títulos.

El proceso de Bolonia, a pesar de que los estados firmantes han ampliado el plazo hasta 2020 para la consecución de algunos objetivos aún no alcanzados, cerraba una primera etapa en 2010 con la Declaración de Budapest-Viena, en la cual fue presentado oficialmente el EEES.

\section{LAS COMPETENCIAS, ELEMENTO CLAVE DEL NUEVO SISTEMA}

Como ya se ha sugerido, la reforma del sistema español de educación superior afectó no sólo a la estructura (duración y organización de los estudios) sino también al contenido, ya que los nuevos títulos se articulan ahora en torno a una serie de competencias que los estudiantes deben adquirir. El objetivo básico del EEES se podría decir que es permitir la comparabilidad, compatibilidad y la coherencia entre los sistemas de educación superior europeos facilitando, entre otras cosas, la movilidad personal y profesional de los nuevos titulados. Aún es pronto para hacer una valoración, pero ciertos estudios apuntan a que aún persisten diferencias entre los sistemas educativos europeos que podrían comprometer dicho objetivo (Senent, 2008).

Aunque ya se ha señalado en distintos contextos, por diversos autores y en documentos de distinta finalidad y alcance que las competencias son un concepto confuso, también parece evidente que es uno de los conceptos clave para entender lo que se ha dado en llamar el proceso de Bolonia (p.e., Ayuga-Téllez et al., 2010). Y no lo son sólo, o fundamentalmente, porque las competencias sean un concepto en torno al cual se articulan los nuevos planes de estudio enmarcados en el marco del EEES, sino, sobre todo, porque hacen referencia a un nuevo paradigma de enseñanza universitaria.

Lo que empezó siendo un intento de homogeneizar la estructura de los estudios universitarios de manera que fueran "entendibles" por todos los estados miembros, se está convirtiendo para la mayoría de los países europeos en la búsqueda de un nuevo modelo de enseñanza y aprendizaje en la universidad, que debería poner al estudiante en el centro del proceso educativo. En este sentido, la autonomía y la autogestión del aprendizaje del estudiante deben convertirse en características esenciales de los sistemas educativos. La razón de ser del cambio de modelo educativo responde fundamentalmente a las transformaciones sociales y productivas que se vienen observando desde la segunda mitad del siglo pasado. Como se puede observar en la Tabla 1, Rué (2008) sintetiza algunas de las diferencias fundamentales entre el paradigma hasta ahora dominante y el enfoque por competencias.

Existen distintas definiciones de competencias con distintos matices, aunque en su mayoría se relacionan con lo que el estudiante va a saber, comprender y ser capaz de llevar a la práctica. Se recogen tres concepciones de distintos documentos europeos:

1. En el Marco de Cualificaciones para el EEES, los resultados de aprendizaje, en los que se incluyen las competencias, se consideran los resultados globales del aprendizaje. Estos consisten en "declaraciones de lo que una persona debe conocer, entender y/o ser capaz de hacer al final de un período de aprendizaje".

2. El Marco Europeo de Cualificaciones para el aprendizaje permanente define competencia como aquella "demostrada capacidad para utilizar conocimientos, destrezas y habilidades personales, sociales y metodológicas, en situaciones de trabajo o estudio y en el desarrollo profesional y personal; en el Marco Europeo de Cualificaciones, la competencia se describe en términos de responsabilidad y autonomía".

3. En el proyecto Tuning se describen como "una combinación dinámica de conocimientos, comprensión, habilidades y capacidades. La promoción de estas competencias es el objeto de los programas educativos. Las competencias cobren forma en varias unidades de curso y son evaluadas en diferentes etapas". 
Tabla 1. Rasgos distintivos del enfoque de formación dominante y el de competencias

\begin{tabular}{|l|l|l|}
\hline \multirow{2}{*}{ Ámbito considerado } & \multicolumn{2}{|c|}{ Prioridades } \\
\cline { 2 - 3 } $\begin{array}{l}\text { El referente de la } \\
\text { Formación }\end{array}$ & $\begin{array}{l}\text { La reproducción y aplicación del } \\
\text { conocimiento generado.I+D }\end{array}$ & $\begin{array}{l}\text { La aportación del conocimiento } \\
\text { desarrollado al crecimiento, al desarrollo, } \\
\text { a la innovación. I+D+I }\end{array}$ \\
\hline Foco o eje de la Formación & Los programas académicos. & $\begin{array}{l}\text { Los estudiantes, sus competencias y el } \\
\text { desarrollo de sus funcionalidades } \\
\text { personales. }\end{array}$ \\
\hline $\begin{array}{l}\text { Acceso dominante al } \\
\text { conocimiento }\end{array}$ & $\begin{array}{l}\text { Enfoques transmisivos Lógico- } \\
\text { deductivos. Racionalidad } \\
\text { intelectual. }\end{array}$ & $\begin{array}{l}\text { Enfoques socio-constructivistas. } \\
\text { Importancia de lo emocional, de lo social } \\
\text { y lo cognitivo. Aprender en y sobre la } \\
\text { acción. }\end{array}$ \\
\hline Contextos formativos & $\begin{array}{l}\text { Aulas, reales, virtuales. División } \\
\text { entre tiempos, espacios } \\
\text { académicos y de profesionalidad } \\
\text { aplicada. }\end{array}$ & $\begin{array}{l}\text { Aulas, contextos sociales y } \\
\text { profesionales. Casos, problemas y } \\
\text { situaciones vitales. }\end{array}$ \\
\hline $\begin{array}{l}\text { Concepción del } \\
\text { conocimiento }\end{array}$ & $\begin{array}{l}\text { Dualidad teoría/ práctica. } \\
\text { Prioridad de la abstracción y la } \\
\text { aplicación. Especialización. }\end{array}$ & $\begin{array}{l}\text { Integración teoría y acción práctica. } \\
\text { Importancia de lo contextual. Integración } \\
\text { diversidad de puntos de vista. } \\
\text { Innovación }\end{array}$ \\
\hline $\begin{array}{l}\text { Concepto de logro } \\
\text { Académico }\end{array}$ & Adaptación a la norma. & $\begin{array}{l}\text { Generación de modalidades complejas } \\
\text { de saber. Capacidad de transferencia. }\end{array}$ \\
\hline Evaluación & $\begin{array}{l}\text { Normativa, en relación a lo } \\
\text { transmitido. Sumativa, final. }\end{array}$ & $\begin{array}{l}\text { Criterial, en relación a los desarrollos } \\
\text { alcanzados. De proceso y sumativa final. }\end{array}$ \\
\hline $\begin{array}{l}\text { Orientación de la } \\
\text { formación, (sentido de } \\
\text { agencia de los formadores) }\end{array}$ & $\begin{array}{l}\text { Estandarizada, de acuerdo con } \\
\text { las normativas oficiales. }\end{array}$ & $\begin{array}{l}\text { De acuerdo con las intencionalidades y } \\
\text { habilidades docentes de los que la } \\
\text { proponen. }\end{array}$ \\
\hline $\begin{array}{l}\text { Función central exigida al } \\
\text { estudiante }\end{array}$ & Adaptativa & $\begin{array}{l}\text { Sentido de la propia responsabilización. } \\
\text { Cooperación. Reflexividad. } \\
\text { Autoevaluación. }\end{array}$ \\
\hline
\end{tabular}

Se puede observar cómo la confusión que se mencionaba anteriormente sobre el concepto de competencias parte, incluso, de los organismos oficiales. Esta cuestión será retomada en los resultados y conclusiones. No obstante, algunos autores como por ejemplo Winterton et al. (2006) plantean un modelo que intenta ser una síntesis de distintas tipologías y que aporte algo de consenso (ver Tabla 2). En su tipología unificada de conocimientos, habilidades y competencias (KSCs) hacen una clasificación de los mismos en torno a dos ejes: conceptual/operacional y ocupacional/personal.

En un nuevo paradigma de educación superior, cada una de las áreas señaladas por estos autores tiene su papel en el proceso educativo del estudiante. Por tanto, no sólo los conocimientos sino los hábitos, los comportamientos y las meta-competencias deben ser objeto de la acción educativa. Este trabajo, desde un enfoque comparativo, intenta describir cómo se ha producido esta reestructuración y cambio de paradigma en los estudios universitarios en el caso concreto de los Grados de Maestro en Educación Primaria. Para ello se han estudiado los títulos de 12 universidades, centrando el análisis en los objetivos, competencias y planificación de los mismos.

Tabla 2. Tipología unificada de los Conocimientos, Habilidades y Competencias (KSCs)

\begin{tabular}{|c|c|c|}
\cline { 2 - 3 } \multicolumn{1}{c|}{} & Ocupacional & Personales \\
\hline Conceptual & Competencias cognitivas & Meta-competencias \\
\hline Operacional & $\begin{array}{c}\text { Competencias } \\
\text { funcionales } \\
\text { (hábitos) }\end{array}$ & $\begin{array}{c}\text { Competencias sociales } \\
\text { (actitudes y } \\
\text { comportamientos) }\end{array}$ \\
\hline
\end{tabular}




\section{LOS TÍTULOS DE GRADO EN MAESTRO EN ESPAÑA}

El denominado proceso de Bolonia ha supuesto un nuevo cambio en la organización de las enseñanzas universitarias $\mathrm{y}$, por tanto, en todos los planes de estudio, incluidos los de maestro. Con el EEES se aboga por un cambio en la relación de enseñanza y aprendizaje, cambio que ha de centrarse en tres aspectos básicos: el rediseño de los currículos, una metodología centrada en el aprendizaje de los alumnos (en vez de en la enseñanza del profesor) y el desarrollo de aprendizajes a partir del enfoque de competencias (Rué, 2007, p. 32). Los tres aspectos están íntimamente relacionados.

En este nuevo paradigma, todo título se diseña sobre la base de las competencias que debe alcanzar el perfil profesional correspondiente. El Ministerio de Educación primero formula las competencias básicas mínimas que se deben adquirir en cualquier título de Grado, y luego, cada título de grado propuesto por cada universidad, formula las competencias específicas que deben lograrse. En el caso de los títulos profesionalizadores, como los de maestro, el Ministerio de Educación también fija unas competencias específicas mínimas para esos títulos.

Tal y como se reconoce en el Real Decreto por el que se establece la ordenación de las enseñanzas universitarias, la flexibilidad y la diversidad son los dos principios que guían la regulación de los nuevos planes de estudio, en cuya elaboración, las universidades tienen un gran margen de maniobra. En el modelo anterior, tras la LRU de 1983, los planes de estudio eran objeto de una mayor regulación. Aunque su elaboración correspondía también a las universidades, primero se fijaban las directrices generales comunes, aplicables a todos los planes de estudio conducentes a cualquiera de los títulos universitarios oficiales, y después las directrices generales propias de cada título. Ambas se fijaban a través de reales decretos. El Consejo de Universidades era quien proponía los títulos universitarios oficiales y las directrices generales propias de cada título. Éstas incluían la denominación del título, los objetivos, el perfil profesional del titulado, la estructura cíclica y la duración máxima y mínima de cada ciclo y las materias troncales, así como unos descriptores de los contenidos de las mismas, los créditos de enseñanza teórica y práctica de cada materia troncal y su vinculación a una o varias áreas de conocimiento. A las universidades les correspondía fijar las materias obligatorias y optativas y, por tanto, sus contenidos, número de créditos teóricos y prácticos y el área o áreas de conocimiento a las que se vinculaban.

En el nuevo sistema, no existe una normativa semejante a las anteriores directrices generales propias salvo en el caso de los títulos conducentes a profesiones reguladas, como es el caso del título de maestro de Educación Primaria. E incluso en este caso, cada universidad propone su propio título, con su propia denominación, perfil, objetivos y competencias, su propia estructura de enseñanzas, sus propios contenidos, etc. Para la elaboración de un plan de estudios conducente a un título, cada universidad debe elaborar una memoria de solicitud para la verificación de dicho título, que debe contener los siguientes aspectos: (1) descripción del título, en el que se refleja la denominación, la universidad solicitante y el centro, el tipo de enseñanza y el número de plazas ofertadas, (2) justificación, referentes externos e internos y procedimiento de elaboración, (3) objetivos y competencias, (4) condiciones de acceso y admisión de estudiantes, (5) estructura de las enseñanzas, organización de la movilidad de estudiantes y descripción de los módulos o materias del plan de estudios, (6) personal académico, (7) recursos, materiales y servicios, (8) resultados previstos, (9) Sistema de Garantía de Calidad y (10) calendario de implantación.

Como maestro de Educación Primaria es una profesión regulada, las universidades deben respetar unos requisitos mínimos al elaborar la memoria de verificación. Dichos mínimos, regulados por la Orden ECl/3857/2007, de 27 de diciembre, afectan fundamentalmente a tres aspectos de la memoria: la denominación del título, los objetivos y competencias y, por último, la estructura de las enseñanzas. Aun así, las denominaciones del título varían de una universidad a otra. Actualmente, en el Registro de Universidades, Centros y Títulos encontramos tres denominaciones distintas: (a) Graduado o Graduada en Educación Primaria (26 universidades); (b) Graduado o Graduada en Maestro de (o en) Educación Primaria (11 universidades) y (c) Graduado o Graduada en Magisterio de (o en) Educación Primaria (3 universidades). 
Respecto a las competencias, la Orden ECl/3857/2007, de 27 de diciembre, por la que se establecen los requisitos para la verificación de los títulos universitarios oficiales que habiliten para el ejercicio de la profesión de Maestro en Educación Primaria fija un mínimo de 12 competencias que todos los títulos de maestro deben incluir (ver Tabla 3). En lo que a la estructura del plan de estudios se refiere, se establecen tres grandes módulos de competencias, a los que se les asigna un mínimo de créditos, se fijan los submódulos mínimos que deben contener y se describen otras competencias que dichos submódulos deben contribuir a alcanzar. La estructura modular puede consultarse en la Tabla 4.

Tabla 3. Competencias que deben incluir los títulos de Maestro en Educación Primaria.

Objetivos. Competencias que los alumnos deben adquirir:

1.- Conocer las áreas curriculares de la Educación primaria, la relación interdisciplinar entre ellas, los criterios de evaluación y el cuerpo de conocimientos didácticos en torno a los procedimientos de enseñanza y aprendizaje respectivos.

2.- Diseñar, planificar y evaluar procesos de enseñanza aprendizaje, tanto individualmente como en colaboración con otros docentes y profesionales del centro.

3.- Abordar con eficacia situaciones de aprendizaje de lenguas en contextos multiculturales y plurilingües. Fomentar la lectura y el comentario crítico de textos de los diversos dominios científicos y culturales contenidos en el currículo escolar.

4.- Diseñar y regular espacios de aprendizaje en contextos de diversidad y que atiendan a la igualdad de género, a la equidad y al respeto a los derechos humanos que conformen los valores de la formación ciudadana.

5.- Fomentar la convivencia en el aula y fuera de ella, resolver problemas de disciplina y contribuir a la resolución pacífica de conflictos. Estimular y valorar el esfuerzo, la constancia y la disciplina personal en los estudiantes.

6.- Conocer la organización de los colegios de educación primaria y la diversidad de acciones que comprende su funcionamiento. Desempeñar las funciones de tutoría y de orientación con los estudiantes y sus familias, atendiendo las singulares necesidades educativas de los estudiantes. Asumir que el ejercicio de la función docente ha de ir perfeccionándose y adaptándose a los cambios científicos, pedagógicos y sociales a lo largo de la vida.

7.- Colaborar con los distintos sectores de la comunidad educativa y del entorno social. Asumir la dimensión educadora de la función docente y fomentar la educación democrática para una ciudadanía activa.

8.- Mantener una relación crítica y autónoma respecto de los saberes, los valores y las instituciones sociales públicas y privadas.

9.- Valorar la responsabilidad individual y colectiva en la consecución de un futuro sostenible.

10.- Reflexionar sobre las prácticas de aula para innovar y mejorar la labor docente. Adquirir hábitos y destrezas para el aprendizaje autónomo y cooperativo y promoverlo entre los estudiantes.

11.- Conocer y aplicar en las aulas las tecnologías de la información y de la comunicación. Discernir selectivamente la información audiovisual que contribuya a los aprendizajes, a la formación cívica y a la riqueza cultural.

12.- Comprender la función, las posibilidades y los límites de la educación en la sociedad actual y las competencias fundamentales que afectan a los colegios de educación primaria y a sus profesionales. Conocer modelos de mejora de la calidad con aplicación a los centros educativos.

Tabla 4. Estructura Modular del Plan de Estudios

\begin{tabular}{|l|c|l|}
\hline \multicolumn{1}{|c|}{ Módulos } & Créditos & \multicolumn{1}{c|}{ Submódulos } \\
\hline $\begin{array}{l}\text { Formación } \\
\text { básica }\end{array}$ & 60 & $\begin{array}{l}\text { Aprendizaje y desarrollo de la personalidad } \\
\text { Procesos y contextos educativos } \\
\text { Sociedad, familia y escuela }\end{array}$ \\
\hline & & $\begin{array}{l}\text { Ciencias experimentales } \\
\text { Ciencias sociales } \\
\text { Didáctico y } \\
\text { disciplinar }\end{array}$ \\
& \multirow{2}{*}{100} & $\begin{array}{l}\text { Latemáticas } \\
\text { Lenguas } \\
\text { Educación musical, plástica y visual } \\
\text { Educación física }\end{array}$ \\
\hline Practicum & 50 & $\begin{array}{l}\text { Prácticas en centros escolares } \\
\text { Trabajo fin de grado }\end{array}$ \\
\hline
\end{tabular}


La regulación más detallada, por tanto, es la que atañe a las competencias, pues se describen competencias generales para el título y competencias para cada uno de los submódulos. La estructura del plan de estudios, aunque es objeto de regulación, sigue dejando mucho margen a las universidades, pues: (1) a los submódulos no se les asigna créditos, por lo que su distribución será fijada por las universidades; (2) tampoco se concretan los módulos ni los submódulos en materias, asignaturas 0 áreas de conocimiento, quedando también a discreción de las universidades; (3) el número total de créditos regulados, de alguna manera, por el Ministerio de Educación es de 210, quedando los 30 restantes sin regulación, pudiendo las universidades asignar las competencias que consideren y establecer así sus propios itinerarios de especialización o menciones cualificadoras.

\section{MÉTODO}

En la página web del Registro de Universidades, Centros y Títulos (RUCT), perteneciente al Ministerio de Educación, a fecha de 28 de febrero de 2011, hay 40 títulos de grado publicados en el Boletín Oficial del Estado (BOE) que habilitan para la profesión de Maestro en Educación Primaria en 41 universidades españolas (pues hay un título que es co-impartido por dos universidades), y otros 11 están en proceso de tramitación, lo que suman un total de 51 títulos de grado en 52 universidades. De ellos, 36 títulos son impartidos por universidades públicas y 15 por universidades privadas.

Aunque para futuras investigaciones se intentará tomar en consideración todas las universidades españolas que han implantado (o están en vías de hacerlo) el Grado de Maestro en Educación Primaria, para este trabajo se decidió seleccionar una muestra de 12 universidades. Para realizar dicha selección, se emplearon fundamentalmente tres criterios. En primer lugar, evidentemente sólo se tuvieron en cuenta las universidades que ofertan el Grado de Maestro en Educación Primaria. El segundo requisito fue que dichas universidades tuvieran publicada la creación de dicho título en el BOE, con el fin de tener la seguridad de que las memorias de verificación de sus títulos no vayan a sufrir ninguna modificación fundamental. Por último, se priorizaron las universidades con más visibilidad en base a dos rankings internacionales de universidades en su última edición (año 2010): el Academic Ranking of World Universities (ARWU), elaborado originalmente por la Universidad Jiao Tong de Shangai y en la actualidad por la Shangai Ranking Consultancy y el Ranking Web de Universidades del Mundo, elaborado por el Laboratorio de Cibermetría del Centro de Ciencias Humanas y Sociales del CSIC y basado en la presencia científica en la web.

De las universidades españolas que cumplían los primeros dos criterios (existencia del grado y publicación en el BOE), se eligieron aquéllas que ocupaban las primeras posiciones del ranking ARWU en su última edición o en alguna de sus ediciones anteriores aunque se situasen en posiciones inferiores en el Ranking Web del CSIC, debido al reconocido prestigio e influencia internacionales de dicho ranking. No obstante, el Ranking Web del CSIC, basado en la presencia en la web, permitió ampliar la muestra de 9 a 12 universidades. Por tanto, tomando en consideración todos los criterios mencionados con anterioridad, las universidades analizadas han sido: (1) Universidad Complutense de Madrid (UCM); (2) Universidad Autónoma de Madrid (UAM), (3) Universidad de Valencia (UV), (4) Universidad de Sevilla (US), (5) Universidad de Murcia (UM), (6) Universidad del País Vasco (UPV), (7) Universidad de Salamanca (USAL), (8) Universidad de Valladolid (UVA), (9) Universidad de Oviedo (UNIOVI), (10) Universidad de Vigo (UVIGO), (11) Universidad Jaume I (UJI) y (12) Universidad de Castilla La Mancha (UCLM). El documento que ha servido de base para la comparación entre las distintas universidades ha sido su memoria de verificación del título de Maestro en Educación Primaria presentada a la Agencia Nacional de Evaluación de la Calidad y Acreditación (ANECA).

\section{RESULTADOS}

El análisis de las memorias se ha centrado, en primer lugar, en el perfil del maestro propuesto por las distintas universidades y que, al no ser siempre explícito, ha tenido que ser inferido de los objetivos. 


\section{Análisis del perfil del maestro, los objetivos y las competencias}

Con respecto a la formulación de los objetivos y de las competencias, el primer aspecto llamativo ha sido la confusión terminológica que se ha producido entre ambos. En concreto, tomando como punto de partida la orden ECl/3857/2007, de 27 de diciembre, por la que se establecen los requisitos para la verificación de los títulos universitarios oficiales que habiliten para el ejercicio de la profesión de Maestro en Educación Primaria, se observa que se incluyen los objetivos que los títulos deben cumplir enumerando doce competencias que los estudiantes deben adquirir (ver Tabla 3, p. 6). Esta falta de precisión, desde nuestro punto de vista, ha provocado que algunas universidades las hayan considerado como objetivos y como competencias.

En primer lugar, atendiendo específicamente a los objetivos, las Universidades de Salamanca (USAL), de Valencia (UV) y la Complutense de Madrid (UCM) han optado por incluir estas doce competencias de la orden ECl/3857/2007, de 27 de diciembre. Las universidades restantes han formulado objetivos distintos, basándose para ello en documentos diferentes. Así, en las universidades que han tomando como punto de referencia el Libro Blanco, como por ejemplo la Universidad Jaume I (UJI) o la de Sevilla (US), los objetivos descritos se relacionan con funciones de enseñanza - aprendizaje. Esto es, algunos de sus objetivos serían que el futuro maestro pueda proporcionar la capacidad de organización de la interacción de cada alumno, actuar como mediador para que toda la actividad que se lleve a cabo resulte significativa y estimule el potencial de desarrollo de cada uno de los alumnos en un trabajo cooperativo del grupo, diseñar y organizar proyectos disciplinares e interdisciplinares y funciones de tutoría, orientación y evaluación de los estudiantes. Con algunas diferencias, estos objetivos se contemplan igualmente en las memorias de la Universidad Autónoma de Madrid (UAM) y la de Castilla-La Mancha (UCLM).

Adicionalmente, universidades como la Universidad de Vigo (UVIGO) y la Universidad de Sevilla (US) han tenido en cuenta los objetivos generales de la etapa de Educación Primaria (Artículo 2 de la LEY ORGÁNICA 2/2006, de 3 de mayo), haciendo un mayor énfasis en funciones de tipo transversal. Por tanto, aluden a la importancia del desarrollo de la personalidad y de las capacidades de los alumnos, el respeto de los derechos y libertades, la responsabilidad, la formación para la paz y la preparación para el ejercicio de la ciudadanía. Otras, como la Universidad de Oviedo (UNIOVI), atendiendo al artículo 16 de la citada LEY ORGÁNICA 2/2006, de 3 de mayo, consideran además la formación de profesionales que afiancen el desarrollo personal y bienestar, además de habilidades culturales básicas relativas a la expresión y compresión oral y escrita, cálculo, habilidades sociales, hábitos de trabajo, creatividad, la capacitación para una acción educativa que integre las experiencias y ritmos de aprendizaje de los alumnos y la preparación de profesionales críticos y comprometidos. La investigación educativa como objetivo está igualmente recogida en la Universidad de Sevilla (US) y la Universidad de País Vasco (UPV).

En segundo lugar, se han analizado también las competencias que los alumnos deben adquirir durante el Grado de Maestro en Educación Primaria. Precisamente, éste es el elemento más regulado, lo que se plasma en que la mayoría de las Universidades, exceptuando las citadas anteriormente, se hayan ceñido a las doce competencias establecidas por la Orden ECI/3857/2007 de 27 de diciembre (ver también Tabla 3, p. 5).

Además, la Universidad Autónoma de Madrid (UAM), la Universidad Complutense de Madrid (UCM) y la Universidad de Castilla-La Mancha (UCLM) han incorporado otras competencias a las ya mencionadas. Por ejemplo, la Universidad Autónoma de Madrid (UAM) incluye, entre otras, la importancia de promover el aprendizaje autónomo, la función tutorial, el respecto a las diferencias personales y culturales y la dimensión ética del maestro. Por su parte, la Universidad Complutense de Madrid (UCM) considera la importancia del autoaprendizaje, la capacidad de innovación y divulgación de hallazgos científicos y las leyes de igualdad efectiva de mujeres y hombres; la de igualdad de oportunidades, no discriminación y accesibilidad universal de las personas con discapacidad, y la de Fomento de la Educación y Cultura de la Paz. Finalmente, la Universidad de Castilla - La Mancha (UCLM) incluye competencias relacionadas con valores y práctica profesional, conocimientos y enseñanza. 


\section{Análisis de la planificación de las enseñanzas}

Como ya se ha señalado en la introducción, el Ministerio de Educación español organizó el título de Maestro en Educación Primaria en tres grandes módulos de competencias: 1) Formación Básica; 2) Formación Didáctico-Disciplinar y 3) Practicum (compuesto, a su vez, por las prácticas externas y el trabajo de fin de grado). Estos tres bloques de competencias suman en cada título 210 créditos europeos. Se dejan, por tanto, 30 créditos sin regular, para que las universidades les asignen competencias, los adscriban a las materias que consideren y los organicen como mejor estimen de acuerdo al perfil u objetivos del título. Normalmente, dichos créditos son los correspondientes a la optatividad, a través de los cuales se pueden organizar menciones 0 itinerarios de especialización.

El análisis de las memorias ha mostrado que todas las universidades analizadas han ofertado 60 créditos en el módulo de Formación Básica, el mínimo fijado por el Ministerio. Seguramente porque es múltiplo de 6, ya que las universidades han optado por asignaturas de 6 y 9 créditos en las materias de formación básica. Sin embargo, el Módulo Didáctico - Disciplinar varía entre 99 y 118 créditos. Como se observa en la Tabla 4, la mayoría de las universidades ha intentado situarse en torno a los 100 créditos (con pequeñas variaciones para adaptarse a los créditos que las universidades fijan como mínimos en cada asignatura). Sin embargo, sólo una Universidad de las analizadas, la Jaume I (UJI) ha aumentado dicho módulo hasta los 118 créditos. Este aumento obedece a que la UJI ha primado la obligatoriedad frente a la optatividad renunciando también a la oferta de menciones cualificadoras.

El módulo de Formación Complementaria, que permite a los alumnos conseguir una mención se compone, en 5 de las universidades, de los 30 créditos que quedaban sin regular en el plan de estudios. Por los ajustes realizados en el módulo Didáctico-Disciplinar, las restantes universidades reducen su oferta de optatividad, en algunos casos, a 24 o 27 créditos, por lo que para organizar menciones hay que recurrir a créditos de los otros tres módulos, Formación Básica, DidácticoDisciplinar o Practicum, o se renuncia a organizar menciones, caso de la UJI.

Por último, con respecto al módulo de Practicum, se ha encontrado que hay bastante homogeneidad en el número de créditos asignado, esto es, 50 créditos. Así, la mayoría de las universidades asigna 44 créditos a las Prácticas externas, siendo el número mínimo de créditos 38 y el máximo 48 y 6 créditos al Trabajo de fin de grado, con excepción de la Universidad del País Vasco (UPV) en la que se le han asignado 12 créditos (ver Tabla 5).

Tabla 5. Estructura Modular y créditos asignados

\begin{tabular}{|c|c|c|c|c|c|c|c|c|c|c|c|c|}
\hline & $\overline{U C M}$ & UM & USAL & UVIGO & UVA & UAM & US & UPV & UOVI & UJI & UV & UCLM \\
\hline $\begin{array}{l}\text { Formación } \\
\text { Básica }\end{array}$ & 60 & 60 & 60 & 60 & 60 & 60 & 60 & 60 & 60 & 60 & 60 & 60 \\
\hline $\begin{array}{l}\text { Didáctico } \\
\text { Disciplinar }\end{array}$ & 100 & 100 & 100 & 102 & 102 & 102 & 100 & 100 & 100 & 118 & 99 & 102 \\
\hline $\begin{array}{l}\text { Formación } \\
\text { Complementaria }\end{array}$ & 30 & 30 & 30 & 24 & 27 & 27 & 30 & 38 & 30 & 12 & 30 & 24 \\
\hline Practicum & $\begin{array}{c}50 \\
44 \\
6\end{array}$ & $\begin{array}{c}50 \\
44 \\
6\end{array}$ & $\begin{array}{c}50 \\
44 \\
6\end{array}$ & $\begin{array}{c}54 \\
48 \\
6\end{array}$ & $\begin{array}{c}51 \\
45 \\
6\end{array}$ & $\begin{array}{c}51 \\
45 \\
6\end{array}$ & $\begin{array}{c}50 \\
44 \\
6\end{array}$ & $\begin{array}{l}50 \\
38 \\
12\end{array}$ & $\begin{array}{c}50 \\
44 \\
6\end{array}$ & $\begin{array}{c}50 \\
44 \\
6\end{array}$ & $\begin{array}{c}51 \\
45 \\
6\end{array}$ & $\begin{array}{c}54 \\
48 \\
6\end{array}$ \\
\hline
\end{tabular}

¿Cuántas menciones y qué tipo de menciones ofertan las universidades?

El número de menciones consideradas por las 11 universidades que ofertan esta posibilidad (recordemos que la UJI no alcanza el número de créditos necesarios en optatividad para ello) oscila desde un mínimo de 2 menciones en la Universidad Complutense de Madrid (UCM) hasta las 12 propuestas por la Universidad Autónoma de Madrid (UAM).

Analizando los tipos de menciones, la primera característica a destacar es la continuidad de las que eran tradicionalmente las especialidades del antiguo plan de estudios de Diplomado en 
Maestro de Educación Primaria (i.e., Educación Musical, Educación Física, Lenguas extranjeras, Educación Especial y Audición y Lenguaje, exceptuando las antiguas especialidades de Educación Infantil y Educación Primaria que ahora son titulaciones diferentes). De hecho, en los nuevos Grados de Maestro de Educación Primaria, las cinco menciones más ofertadas son: a) Educación Musical (11 universidades); b) Educación Física (10 universidades); c) Lenguas Extranjeras (10 universidades); d) Educación Especial (10 universidades; nótese que se han considerado las nomenclaturas de menciones tales como Necesidades Específicas de apoyo educativo y apoyo a la diversidad, Apoyo a la inclusión educativa o Educación inclusiva); y e) Audición y Lenguaje (4 universidades).

Sin embargo, la posibilidad de incorporar nuevos caminos en la profesión de maestro se ha hecho notar en la diversidad de menciones en las propias universidades y entre ellas. Por ejemplo, 3 universidades ofertan menciones en Lengua castellana y literatura, en Matemáticas, en Entorno, naturaleza y sociedad y en Orientación y Tutoría. Igualmente, 2 universidades incorporan las menciones en Nuevas Tecnologías de la Información, en Arte, en Profundización curricular y en Educación Intercultural. Por último, los alumnos que deseen cursar menciones en Lengua de Signos, en Lengua asturiana, en Lengua Euskara y en Educación Religiosa Escolar sólo podrán hacerlo en una única universidad.

\section{Practicum ¿Qué modelo se ha adoptado y cómo se ha distribuido a lo largo del Grado?}

Como ya se ha comentado, el módulo de Practicum se compone de Prácticas externas y Trabajo de fin de grado, comno se muestra en la Tabla 6). En esta tabla, no se encuentra publicado el número de créditos que la Universidad de Murcia asigna al Practicum de $2^{\circ}, 3^{\circ}$ y $4^{\circ}$ curso. Si atendemos, en primer lugar, a las Prácticas Externas, se diferencian claramente dos modelos posibles. El primero de ellos consiste en ubicar las prácticas en distintos cursos académicos, de modo que éstas sean una actividad educadora que contribuya igualmente a la adquisición de las competencias. Este modelo ha sido adoptado mayoritariamente (i.e, 11 de las 12 universidades). La distribución de los créditos por cursos se ha hecho de la siguiente forma: i) En dos cursos académicos: aproximadamente $18-20$ créditos en tercero y 24 créditos en cuarto (5 universidades); y ii) En tres cursos académicos (6 universidades). El más breve, que comprende aproximadamente entre 8-9 créditos, se cursa durante el segundo año (excepto en la Universidad de Valencia (UV) que se comienza en el segundo semestre del primer año), un segundo periodo de mayor duración en el tercer curso de grado y, por último, el periodo de prácticas más largo se reserva para el cuarto año.

El segundo modelo consiste en concentrar todo el módulo de Practicum, Prácticas externas y Trabajo de fin de grado, en el último curso académico. Este modelo ha sido adoptado por la Universidad de Vigo (UVIGO).

Tabla 6. Distribución del practicum por cursos y número de créditos asignados

\begin{tabular}{|c|c|c|c|c|c|c|c|c|c|c|c|c|}
\hline & UCM & UM & USAL & UVIGO & UVA & UAM & US & UPV & UOVI & UJI & UV & UCLM \\
\hline $1^{\circ}$ & & & & & & & & & & & 6 & \\
\hline $2^{\circ}$ & 8 & -- & & & & 10 & & 9 & 8 & & & \\
\hline $3^{\circ}$ & 6 & -- & 20 & & 20 & 12 & 30 & 11 & 12 & 18 & 16.5 & 18 \\
\hline $4^{\circ}$ & 30 & -- & 24 & 48 & 24 & 27 & 14 & 18 & 24 & 26 & 22.5 & 24 \\
\hline
\end{tabular}

\section{CONCLUSIONES}

1.- Las competencias, si bien son un concepto confuso que ni siquiera está suficientemente consensuado desde los propios organismos oficiales, son el elemento fundamental en torno al cual se articulan los nuevos planes de estudio en el marco del EEES. Incluso en la normativa ministerial se refleja dicha confusión cuando se habla indistintamente de objetivos y competencias, sin hacer distinción entre ambos conceptos. 
2.- La flexibilidad y la diversidad son los dos principios que guían la regulación de los nuevos planes de estudio, en cuya elaboración las universidades tienen un gran margen de maniobra. Cada universidad propone su propio título, con su propia denominación, perfil, objetivos y competencias, su propia estructura de enseñanzas, sus propias materias y sus propios contenidos.

3.- Como maestro de educación primaria es una profesión regulada, las universidades deben atenerse a unos requisitos mínimos en la elaboración de sus planes de estudios, mínimos que afectan fundamentalmente a tres aspectos: denominación del título, objetivos y competencias y, por último, estructura de las enseñanzas.

4.- La regulación más detallada es la que atañe a las competencias, descritas en la orden $\mathrm{ECl} / 3857 / 2007$ de 27 de diciembre, verdaderos elementos aglutinadores de las memorias estudiadas. Precisamente aquí es donde se produce la confusión entre objetivos y competencias del título de Educación Primaria. La planificación de las enseñanzas, es decir, la adscripción de las competencias a materias y asignaturas y su secuenciación temporal, está menos regulada, siendo la parte en la que más diferencias aparecen.

5.- Las competencias no tradicionales, como por ejemplo la autonomía, el liderazgo o el trabajo en equipo no se han regulado por la orden ECl/3857/2007 de 27 de diciembre. Sólo algunas de ellas figuran en el REAL DECRETO 1393/2007, de 29 de octubre, por el que se establece la ordenación de las enseñanzas universitarias oficiales y, en contadas ocasiones, se incluyen en las memorias analizadas. Sin embargo las competencias genéricas cobran especial importancia sobre todo en escenarios turbulentos como el actual, en el que los empleadores y las instituciones más que insistir en una demanda específica de conocimientos de disciplinas tradicionales dan cada vez más importancia a lo transversal, como los conocimientos de idiomas, la iniciativa, la capacidad de adaptación, etcétera (Alonso, Fernández Rodríguez y Nyssen, 2009).

6.- La especificidad de los distintos estudios de Grado de Maestro en Educación Primaria se observa, principalmente, en el modelo de practicum adoptado y la cantidad y tipo de menciones o materias optativas ofertadas. También resulta significativa la distinta adscripción a materias de las competencias, es decir, el distinto peso que cada universidad da a las distintas materias en el plan de estudios, si bien esto sería para una investigación posterior.

\section{REFERENCIAS}

Alonso, L. E., Fernández Rodríguez, C. J. y Nyssen, J. M., El debate sobre las competencias. Una investigación cualitativa en torno a la educación superior y el mercado de trabajo en España, $1^{a}$ edición. ANECA, Madrid, España (2009).

Aneca. Libro Blanco. Título de Grado en Magisterio. Vol. 1 (en línea), 2004. http://www.aneca.es/var/media/150404/libroblanco_jun05_magisterio1.pdf Acceso: 15 de marzo (2010).

Ayuga Téllez, E., González García, C. y Grande Ortiz, M. A., Análisis de competencias en el Grado de Ingeniería Forestal para su adaptación al Espacio Europeo de Educación Superior, Form. Univ., 3(3), 3-14 (2010).

Fernández Díaz, M. J., Carballo Santaolalla, R. y Galán González, A., Faculty attitudes and training needs to respond the new European Higher Education challenges, Higher Education, 60, 101-118 (2010).

Ley Orgánica 11/1983, de 25 de agosto, de Reforma Universitaria (LRU) (BOE 209/1983 de 1 de septiembre de 1983).

Ley Orgánica 2/2006, de 3 de mayo, de Educación (BOE 106/2006 de 4 de mayo de 2006). 
Ley Orgánica 4/2007, de 12 de abril, de Modificación de la Ley Orgánica de Universidades (LOMLOU) (BOE 89/2007 de 13 de abril de 2007)

Ley Orgánica 6/2001, de 21 de diciembre, de Universidades (LOU) (BOE 307/2001 de 24 de diciembre de 2001).

Ministerio de Ciencia, Tecnología e Investigación. A framework for qualifications for the European Higher Education Area, (en línea), 2005. http://www.bologna-bergen2005.no/Docs/00Main_doc/050218_QF_EHEA.pdf Acceso: 28 de febrero (2011).

Orden ECl/3857/2007, de 27 de diciembre, por la que se establecen los requisitos para la verificación de los títulos universitarios oficiales que habiliten para el ejercicio de la profesión de Maestro en Educación Primaria (BOE 312/2007 de 29 de diciembre de 2007).

Real Decreto 1393/2007, de 29 de octubre, por el que se establece la ordenación de las enseñanzas universitarias oficiales (BOE 260/2007 de 30 de octubre de 2007).

Real Decreto 1440/1991, de 30 de agosto, por el que se establece el título universitario oficial de Maestro, sus diversas especialidades y las directrices generales propias de los planes de estudios conducentes a su obtención (BOE 24/91 de 11 de octubre de 1991).

Rué, J., Enseñar en la Universidad. El EEES como reto para la Educación Superior, $1^{\mathrm{a}}$ edición. Narcea, Madrid, España (2007).

Rué, J., Formar en competencias en la universidad: entre la relevancia y la banalidad. Red U. Revista de Docencia Universitaria, número monográfico 1 "Formación centrada en competencias", 1-19 (2008).

Senent, J. M., Las titulaciones de educación: una perspectiva histórico-comparada en España y Europa, Educación XXI, 11, 43-71 (2008).

Teichler, U., Sistemas comparados de educación superior en Europa. Marcos conceptuales, resultados empíricos y perspectiva de futuro, $1^{\text {a }}$ edición. Octaedro, Barcelona, España (2009)

Tuning Educational Structures in Europe, (en línea), 2007. http://tuning.unideusto.org/tuningeu Acceso: 28 de febrero (2011).

Winterton, J., Delamare, F. y Stringfellow, E., Typology of Knowledge, Skills and Competences: clarification of the concept and prototype, (en línea), 2006. CEDEFOP Reference Series, 64, Luxembourg, Office for Official Publications of the European Communities. http://www.evta.net/website_docs/typologiesofksc_2011.pdf Acceso: 20 de octubre (2011). 